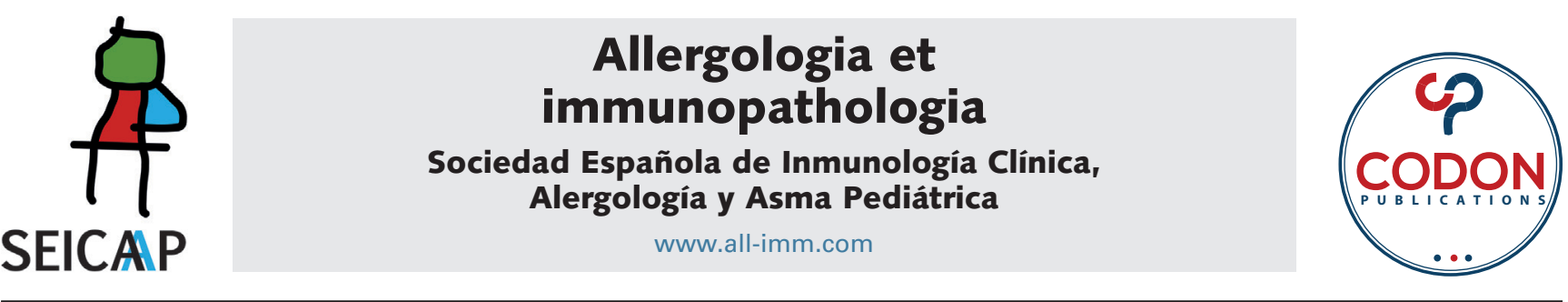

ORIGINAL RESEARCH

OPEN ACCESS (c) (1) (2)

\title{
SEMA3A protects against hyperoxia-induced lung injury in a bronchopulmonary dysplasia model of newborn rat by inhibiting ERK pathway
}

\author{
Zhenyu Liang, Xiao Zhang, Yingxian Liu, Qianmei Wu, Chuming You*
}

Department of Pediatrics, Guangdong Second Provincial General Hospital, Guangdong Province, China

Received 9 July 2021; Accepted 21 August 2021

Available online 1 November 2021

\author{
KEYWORDS \\ apoptosis; \\ bronchopulmonary \\ dysplasia; \\ ERK/JNK; \\ hyperoxia; \\ inflammation; \\ lung injury; \\ SEMA3A
}

\begin{abstract}
Background: Hyperoxia induces lung injury through lung inflammation in premature infants, leading to bronchopulmonary dysplasia (BPD). Semaphorin 3A (SEMA3A) participates in diverse biological processes, including cell migration, angiogenesis, and inflammation. The effect of SEMA3A on hyperoxic lung injury of neonatal rats with BPD was investigated in this study.

Methods: Neonatal rats with BPD were established through hyperoxia treatment. Hematoxylineosin staining was used to evaluate histopathological analysis in lung tissues. SEMA3A expression was assessed by reverse transcription-quantitative polymerase chain reaction (RT-qPCR) and western blot assay. Adeno-associated virus (AAV)-mediated over-expression of SEMA3A (AAV-SEMA3A) was administrated into hyperoxia-induced rats, and apoptosis was evaluated by TUNEL staining. Levels of inflammatory cytokines were investigated by enzyme-linked-immunosorbent serologic assay (ELISA).

Results: Hyperoxia-induced histopathological changes in lung tissue reduced alveolar number and enhanced alveolar interval and alveolar volume. SEMA3A was downregulated in lung tissue of hyperoxia-induced rats. AAV-SEMA3A injection attenuated hyperoxia-induced cell apoptosis in lung tissues by increasing $\mathrm{Bcl}-2$ and decreasing Bax and cleaved caspase-3. Moreover, the enhanced levels of Interleukin (IL)-1 $\beta$, monocyte chemoattractant protein (MCP)-1, and tumor necrosis factor- $\alpha$ (TNF- $\alpha$ ) in hyperoxia-induced rats were restored by AAV-SEMA3A injection by the downregulation of nuclear factor kappa $B$ (NF-kB) phosphorylation. AAV-SEMA3A injection also ameliorated histopathological changes in lung tissues of hyperoxia-induced rats by increasing the number of radial alveolar count and decreasing the volume of mean linear intercept. Besides, the protein expression levels of extracellular signal-regulated kinase (ERK) and c-Jun N-terminal kinase (JNK) phosphorylation were reduced in hyperoxia-induced rats post-AAV-SEMA3A injection. Conclusion: Ectopical expression of SEMA3A suppressed hyperoxia-induced apoptosis and inflammation in neonatal rats, and ameliorated the histopathological changes through inactivation of ERK/JNK pathway.
\end{abstract}

(C) 2021 Codon Publications. Published by Codon Publications.

*Corresponding author: Chuming You, Department of Pediatrics, Guangdong Second Provincial General Hospital, No. 466 Xingang Zhong Lu, Haizhu District, Guangzhou City 510317, Guangdong Province, China. Email address: youchuming@163.com 


\section{Introduction}

Bronchopulmonary dysplasia (BPD), a common lung disease in premature infants, is caused by physiological lung dysplasia. ${ }^{1}$ BPD is an important cause of respiratory diseases in preterm newborns, leading to significant morbidity and mortality. ${ }^{2}$ The main characteristics of BPD are enlarged alveoli, pulmonary growth arrest, mesenchymal cell hyperplasia, abnormal lung function, and fibrosis. ${ }^{3}$ Preterm birth, oxygen toxicity, postnatal infection, inflammation, and prenatal infection are considered to be closely related to the progression of BPD ${ }^{4}$ Treatment strategies, such as diuretics and glucocorticoids, demonstrated limited clinical efficiency in the treatment of BPD. ${ }^{5}$ Therefore, novel treatments are urgently required for BPD.

Although mechanical oxygen supply is one of the key methods for treating BPD, hyperoxia induces severe adverse effects in lung tissue, such as alveolar vascular cell permeability, alveolar epithelial and endothelial cell death. ${ }^{6}$ Preterm infants are susceptible to hyperoxia, as it induces excessive accumulation of reactive oxygen species, and activates pathways to promote the secretion of inflammatory factors in alveolar epithelial cells, thus promoting inflammation and alveolar development deficits in neonatal rats, similar to the features found in infants with BPD. ${ }^{7}$ Moreover, hyperoxia-induced apoptosis of lung epithelial cells is another prominent feature of hyperoxiainduced acute lung injury. ${ }^{8}$ Therefore, strategies that alleviate hyperoxia-induced inflammation and apoptosis of lung tissue are widely investigated in the prevention of BPD. ${ }^{9}$

Class 3 semaphorins, including seven members (3A$3 G)$, are secreted proteins in vertebrates that regulate lymph angiogenesis, angiogenesis, immune responses, and other physiological and developmental functions. ${ }^{10}$ SEMA3A has been found to exert anti-cancer and anti-inflammatory properties. For example, the proliferation of brain tumor stem cells of glioblastoma multiform was suppressed by SEMA3A. ${ }^{11}$ Silence of SEMA3A promoted tumor cell migration and invasion through activation of mitogen-activated protein kinase (MAPK) cascade. ${ }^{12}$ SEMA3A alleviated osteoarthritis by inhibiting the inflammatory response of chondrocytes to excessive mechanical stress. ${ }^{13}$ Radiation-induced osteoporosis was also attenuated by SEMA3A through reduction of inflammation and inhibition of osteoclasts. ${ }^{14}$ In respiratory system, loss of SEMA3A altered distal lung structure, and was associated with increased alveolar septal cell death in neonatal mice, suggesting that SEMA3A plays an important role in regulating development of the lung and alveolar separation. ${ }^{15}$ Moreover, deficiency of SEMA3-neuropilin pathway thickens alveolar septa, misaligns pulmonary veins, and reduces capillary density and pulmonary surfactant secretion, thus leading to atelectatic and immature regions in the lungs; all this suggests the clinical relevance of SEMA3 in neonatal respiratory disorders. ${ }^{16}$ However, the protective effect of SEMA3A against hyperoxia-induced BPD has not been reported yet.

In this study, the expression of SEMA3A in lung tissue of hyperoxia-induced neonatal rats was evaluated, and the effects of SEMA3A on cell apoptosis and inflammation of lungs were explored. The results might provide a novel strategy for preterm infants with hyperoxiainduced BPD.

\section{Materials and Methods}

\section{Animals}

This study was approved by the Medical Ethics Committee of Guangdong Second Provincial General Hospital (Approval No. 2021-KZ-028-02) in accordance with the Guidance for the Care and Use of Laboratory Animals. A total of 24 neonatal Wistar rats were purchased from the Animal Center of the Chinese Academy of Science (Shanghai, China), and maintained with breastfeeding. Rats were divided into two groups: control $(\mathrm{N}=6)$ and BPD $(\mathrm{N}=18)$. For establishing BPD model, the neonatal rats were exposed to atmosphere with continuous $90 \% \mathrm{O}_{2}$ for 7 days. The control group rats were maintained in normal atmosphere. Rats in the BPD group were further divided into 3 subgroups: BPD $(N=6)$, BPD+AAV-Scramble $(N=6)$, and BPD+AAV-SEMA3A $(N=6)$. AAV-Scramble and AAV-SEMA3A were purchased from Genepharma (Shanghai, China). The BPD rats were injected with AAV-Scramble or AAV-SEMA3A virus $\left(8 \times 10^{11} \mathrm{VG} /\right.$ rat $)$ via tail vein on 7 th day of post-hyperoxia induction. After 14 days of hyperoxia induction, rats were anesthetized with $80 \mathrm{mg} / \mathrm{kg}$ body weight sodium pentobarbital injection. Lung tissues were harvested for functional assays, and the animals were sacrificed by cervical dislocation.

\section{Histopathology of lung tissues}

Lung tissues were isolated from each rat, and fixed in $4 \%$ paraformaldehyde. The paraffin-embedded tissues were cut into $6-\mu \mathrm{m}$ sections. The sections were stained with hematoxylin-eosin (H\&E) staining (Solarbio Science \& Technology, Beijing, China) and measured under light microscopy (Nikon, Tokyo, Japan). Line was drawn from the center of the peripheral bronchiole to the nearest connective tissue septum, and the number of alveoli along the line was counted; this was viewed as radial alveolar count (RAC). Five lines in each field were drawn, and the mean linear intercept (MLI) was calculated as length of each line divided by the number of alveolar intercepts.

\section{Terminal deoxynucleotidyl transferase dUTP nick end labeling (TUNEL) and enzyme-linked- immunosorbent serologic assay (ELISA)}

The sections isolated from lung tissue were de-paraffinized and rehydrated in an ethanol series. Sections were incubated with proteinase K (Sigma-Aldrich, Stockholm, Sweden), and treated with TUNEL reaction mixture (Roche Applied Science, Pennsburg, Germany) to determine TUNEL-positive cells under light microscopy (Nikon). Lung tissues were lysed in radioimmunoprecipitation assay lysis buffer (Beyotime, Shanghai, China), and supernatants were collected for ELISA analysis of Interleukin (IL)-1 $\beta$, monocyte chemoattractant protein (MCP)-1, and tumor necrosis factor (TNF)- $\alpha$ using corresponding commercial kits (Dakewe, Shenzhen, China). 
Reverse transcription-quantitative polymerase chain reaction ( $R T-q P C R)$

RNAs were isolated from lung tissue by Trizol (Invitrogen, Carlsbad, CA, USA). RNAs were transcribed into complementary DNA (CDNAs), and RT-qPCR analysis of SEMA3A was performed by SYBR Premix Ex Taq II (TaKaRa, Tokyo, Japan). GAPDH was used as an endogenous control, and the expression level of SEMA3A was calculated using $2^{-\triangle \Delta C q}$. The primers used are as follows: SEMA3A (forward primer: 5'-ACCCAACTATCAATGGGTGCCTTA-3' and reverse primer: 5'-AACACTGGATTGTACATGGCTGGA-3') and GAPDH (forward primer: 5'-CCATCTTCCAGGAGCGAGAT-3' and reverse primer: 5'-TGCTGATGATCTTGAGGCTG-3').

\section{Western blot assay}

Proteins $(40 \mu \mathrm{g})$ from lysates of the lung tissue were separated by SDS-PAGE, and transferred to PVDF membranes (Millipore, Shanghai, China). The membranes were blocked in PBST with $5 \%$ nonfat milk, and incubated with primary and secondary antibodies, including anti-cleaved caspase-3, anti-c-Jun N-terminal kinase (JNK) and anti-p-JNK (1:2000; Abcam, Cambridge, UK), anti-SEMA3A and anti- $\beta$-actin (1:2500; Abcam), anti-Bax and anti-Bcl2 (1:3000; Abcam), anti-NF-kB and anti-p-NF-kB (1:3500; Cell Signaling, Boston, $M A, U S A)$, and anti-extracellular signal-regulated kinase 1/2 (ERK1/2) and anti-p-ERK1/2 (1:4000; Abcam). ECL reagents (Roche, Shanghai, China) were used to detect protein signals.

\section{Statistical analysis}

All the experimental data were depicted as mean \pm standard deviation (SD). Differences between groups were analyzed by Student's $t$-test or One-Way Analysis of Variance using SPSS 11.0 software. Independent sample $t$-test was used for comparison between the two groups if assumptions of normality and homogeneity of variance were satisfied, otherwise the nonparametric Mann-Whitney test was used for analysis; $\mathrm{P}<0.05$ was considered as statistically significant.

\section{Results}

SEMA3A was downregulated in hyperoxia-induced $B P D$ rats

The neonatal rats were administrated with hyperoxia to establish rat models of BPD. Histopathological changes in lung tissue caused by hyperoxia were examined by $\mathrm{H} \& \mathrm{E}$ staining (Figure 1A). As depicted in Figure 1A, hyperoxia induced severe lung injuries, including inflammatory cell infiltration, reduced alveolar number, and enhanced alveolar interval and alveolar volume in BPD rats. Besides, SEMA3A was downregulated in the lung tissue isolated from hyperoxia-induced rats compared to the control (Figures $1 \mathrm{~B}$ and $1 \mathrm{C})$.

\section{SEMA3A suppressed hyperoxia-induced cell} apoptosis in BPD rats

To investigate the role of SEMA3A in the progression of BPD, hyperoxia-induced rats were injected with AAV-SEMA3A for over-expression of SEMA3A. Rats in the BPD group demonstrated lower body weight, lung weight, and decreased lungto-body weight ratio than that in the control group (Table 1). However, AAV-SEMA3A injection given to rats of the BPD group restored decreased body weight, lung weight, and lung-to-body weight ratio (Table 1). AAV-SEMA3A injection upregulated the protein expression of SEMA3A (Figure 2A). Over-expression of SEMA3A also attenuated hyperoxia-induced cell apoptosis in BPD rats (Figure 2B), evidenced by the reduced TUNEL-positive cells (Figure $2 \mathrm{C}$ ). Moreover, the reduced expression level of $\mathrm{Bcl}-2$, enhanced expression levels of Bax, and cleaved caspase- 3 in BPD rats were restored by the over-expression of SEMA3A (Figure 2D), suggesting the anti-apoptotic role of SEMA3A in BPD rats.

\section{SEMA3A suppressed hyperoxia-induced inflammation in BPD rats}

The levels of IL-1 $\beta, M C P-1$, and TNF- $\alpha$ were increased in hyperoxia-induced BPD rats (Figure $3 \mathrm{~A}$ ), while AAV-SEMA3A injection decreased the levels of IL-1 $\beta, M C P-1$, and TNF- $\alpha$ (Figure 3A). The protein expression of NF-kB phosphorylation ( $\mathrm{p}-\mathrm{NF}-\mathrm{kB})$ was enhanced in hyperoxia-induced BPD rats (Figure $3 \mathrm{~B}$ ), suggesting that hyperoxia promoted the activation of NF-kB to induce inflammation. However, over-expression of SEMA3A reduced the level of $\mathrm{p}-\mathrm{NF}-\mathrm{kB}$ in hyperoxia-induced rats (Figure $3 B$ ), revealing the antiinflammatory role of SEMA3A against progression of BPD.

\section{SEMA3A ameliorated hyperoxia-induced histopathological changes in BPD rats}

$B P D+A A V-S E M A 3 A$ group established decreased inflammatory cell infiltration, alveolar interval, and alveolar volume as well as enhanced alveolar number in the lung tissue (Figure 4), thus demonstrating that SEMA3A ameliorated hyperoxia-induced alveolar injury, simplification, and inflammation, which reduced lung injury. Moreover, hyperoxia-induced delayed alveolar development, reduced alveolar diameter (MLI), and enhanced volume of alveolar intercept (RAC) were ameliorated by the over-expression of SEMA3A (Figure 4).

\section{SEMA3A suppressed the activation of ERKIJNK pathway in BPD rats}

The protein expressions of ERK and JNK were not significantly affected by hyperoxia treatment or AAV-SEMA3A injection (Figure 5). However, the expression levels of ERK and JNK phosphorylation were upregulated by hyperoxia treatment (Figure 5). Moreover, AAV-SEMA3A injection downregulated the expression levels of ERK and JNK phosphorylation (Figure 5), indicating that SEMA3A might suppress the activation of ERK/JNK pathway to attenuate progression of BPD. 
(A)
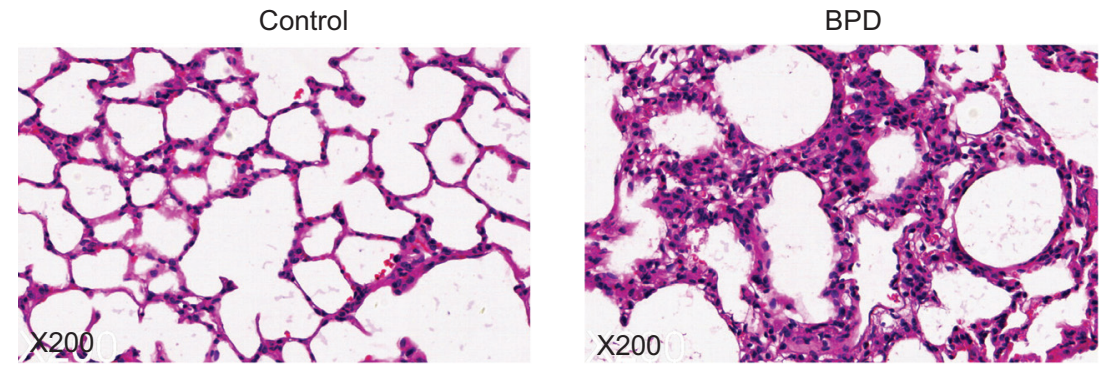

(B)

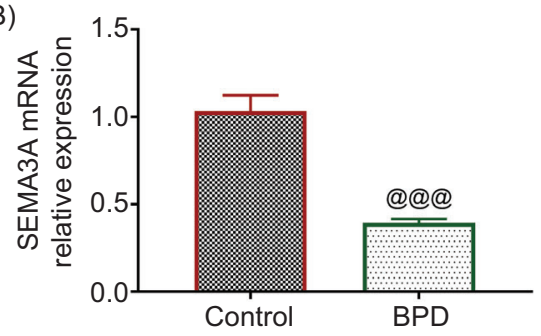

(C)
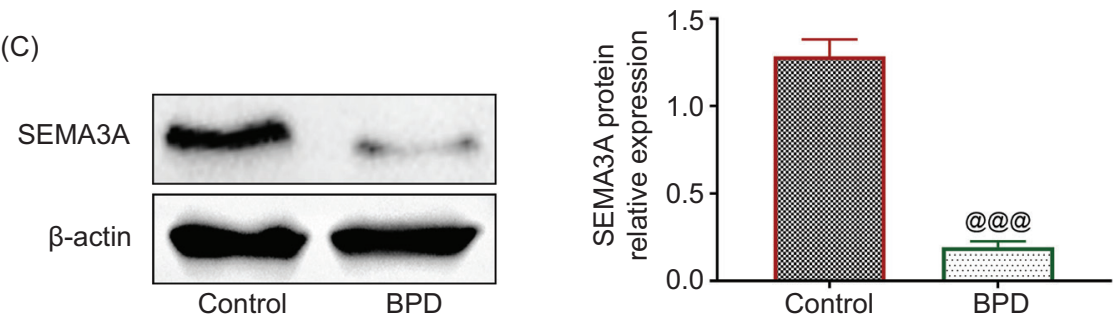

Figure 1 SEMA3A was downregulated in hyperoxia-induced BPD rats. (A) Hematoxylin-eosin staining established that hyperoxia induced severe lung injuries, including inflammatory cell infiltration, reduced alveolar number, and enhanced alveolar interval and alveolar volume in BPD rats. (B) The mRNA expression of SEMA3A was reduced in lung tissue isolated from hyperoxia-induced rats compared to the control group. (C) The protein expression of SEMA3A was reduced in lung tissue isolated from hyperoxia-induced rats compared to the control group. @@@ vs. control, P < 0.001. BPD: bronchopulmonary dysplasia; SEMA3A: semaphorin 3A.

Table 1 Basic characteristics of rats in each group.

Group $n$ Body weight Lung weight Lung-to-body

(g)

(g) weight ratio $(\%)$

\begin{tabular}{lrrrr}
\hline Control & 6 & $13.06 \pm 1.14$ & $0.29 \pm 0.04$ & $2.25 \pm 0.24$ \\
BPD & 6 & $10.42 \pm 0.64^{* *}$ & $0.17 \pm 0.03^{* *}$ & $1.59 \pm 0.30^{*}$ \\
& & & \\
$\begin{array}{l}\text { BPD+AAV- } 6 \\
\text { Scramble }\end{array}$ & $10.29 \pm 0.54$ & $0.16 \pm 0.03$ & $1.50 \pm 0.22$ \\
$\begin{array}{l}\text { BPD+AAV- } 6 \\
\text { SEMA3A }\end{array}$ & $11.94 \pm 0.93^{\dagger}$ & $0.24 \pm 0.03^{\dagger \dagger}$ & $2.06 \pm 0.33^{\dagger}$ \\
\hline
\end{tabular}

Values are presented as mean \pm standard deviation (SD). " $P<0.01{ }^{\text {"* }} \mathrm{P}<0.001$ compared with the Control.

${ }^{+} \mathrm{P}<0.05{ }^{{ }^{+t} \mathrm{P}}<0.01$ compared with the BPD+AAV-Scramble.

\section{Discussion}

SEMA3 binds to neuropilin receptors and recruits plexin family to participate in the process of pulmonary vascular development, and the disruption of pulmonary vascular morphogenesis is implicated in the pathogenesis of pulmonary diseases in infancy, including BPD. ${ }^{17}$ SEMA3C was reportedly reduced in the lungs of $\mathrm{O}_{2}$-induced newborn rats, and treatment with SEMA3C attenuated hyperoxia-induced decreased viability of alveolar epithelial cells, promoted wound healing, and preserved the lung and alveolar vascular growth, thus ameliorating progression of BPD. ${ }^{18}$ Considering the association between SEMA3A and alveolar septal cell death in neonatal mice,$^{15}$ the protective effect of SEMA3A against hyperoxia-induced BPD was investigated in this study.

Hyperoxia has been demonstrated to induce reduction in dynamic lung compliance and enhancement in airway resistance, thus impairing lung function. ${ }^{5}$ Therefore, hyperoxia-induced oxygen toxicity was widely used to establish BPD model in newborn rats. ${ }^{5}$ In this study, prevention of alveolarization, and simpler and wider alveoli formation, was observed in the lungs of hyperoxia-induced neonatal rats, thus demonstrating hyperoxia-induced lung injury in newborn rats. SEMA3A was identified to be downregulated in the lung tissue of hyperoxia-induced neonatal rats, suggesting that SEMA3A could be involved in the development of BPD. AAV-mediated over-expression of SEMA3A ameliorated hyperoxia-induced histopathological changes in rats 
(A)

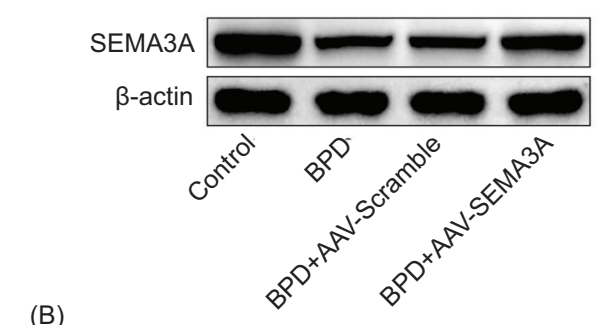

(B)
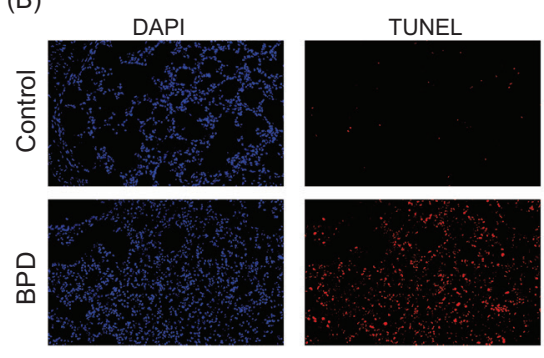

(D)

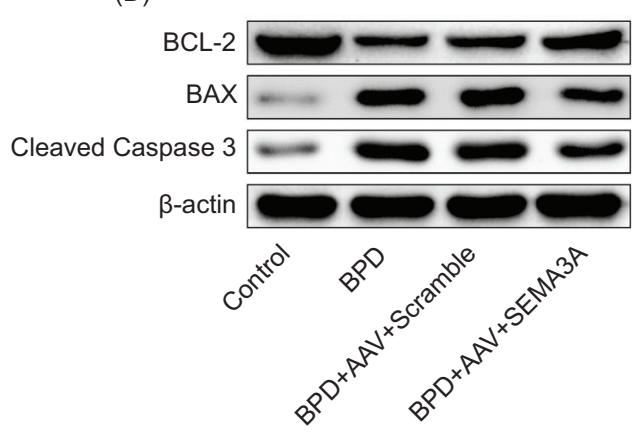

(C)

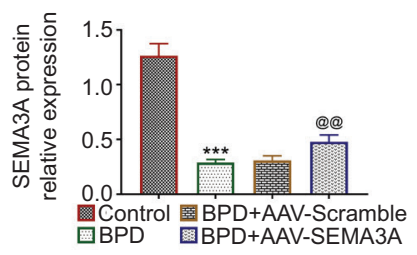

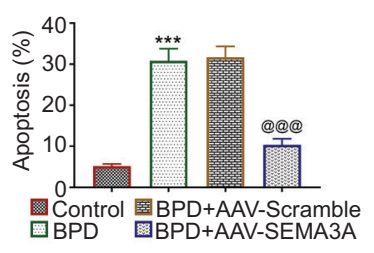
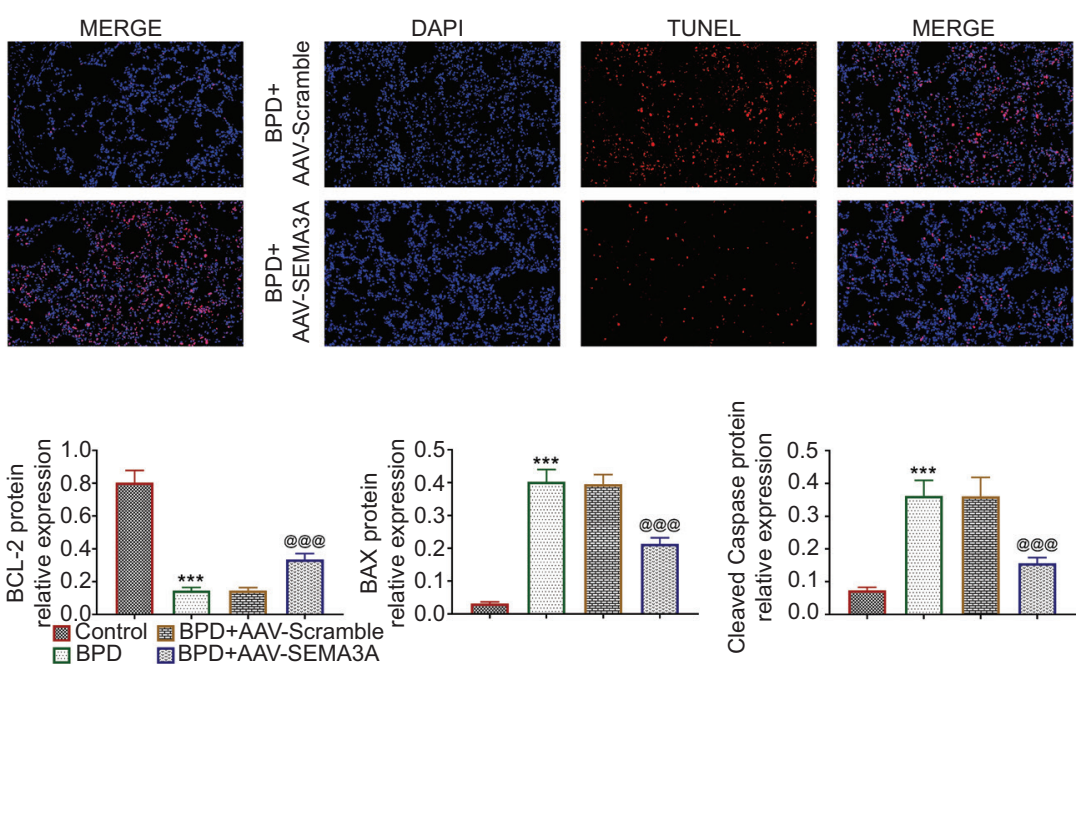

Figure 2 SEMA3A suppressed hyperoxia-induced cell apoptosis in BPD rats. (A) AAV-SEMA3A injection promoted the protein expression of SEMA3A. (B) AAV-SEMA3A injection attenuated hyperoxia-induced cell apoptosis in BPD rats. (C) AAV-SEMA3A injection attenuated hyperoxia-induced increased number of TUNEL-positive cells in BPD rats. (D) AAV-SEMA3A injection attenuated hyperoxia-induced reduced expression of $\mathrm{Bcl}-2$, and enhanced expression levels of Bax and cleaved caspase-3 in BPD rats. ${ }^{* * *}$ vs. control, $\mathrm{P}<0.001$. ${ }^{\circledR}$, @@@ vs. BPD+AAV-Scramble, $\mathrm{P}<0.01, \mathrm{P}<0.001$. BPD: bronchopulmonary dysplasia; SEMA3A: semaphorin 3A.

by enhancing the count of RAC and reducing the volume of $M L I$, thereby confirming the protective effect of SEMA3A against BPD.

Apoptosis is a common manifestation of hyperoxia-induced lung injury. ${ }^{19}$ Suppression of cell apoptosis is regarded as a potential therapeutic strategy for the treatment of hyperoxia-induced lung injury in BPD. ${ }^{19}$ SEMA3A induced pro-apoptotic program in ischemia-reperfusion-induced acute kidney injury, ${ }^{20}$ and repression of SEMA3A protected retinal ganglion cell against the optic nerve crush-induced apoptosis. ${ }^{21}$ However, in this study, over-expression of SEMA3A decreased cell apoptosis through upregulation of Bcl-2 and downregulation of Bax and cleaved caspase-3 in hyperoxia-induced neonatal rats, indicating the antiapoptotic role of SEMA3A in BPD. The pathway involved in SEMA3A-mediated cell apoptosis in hyperoxia-induced BPD must be investigated in the future research. Moreover, alveolar simplification and reduced pulmonary vessel density were considered as typical histological patterns of BPD. ${ }^{18}$ A previous study has indicated that promotion of alveolar epithelial cell proliferation by SEMA3C attenuated hyperoxia-induced lung injury. ${ }^{18}$ Therefore, the effect of SEMA3A on alveolar epithelial cell proliferation could be helpful to confirm its protective effect against BPD. Functions of airway epithelial cells are important for lung homeostasis, and differential functional activities of airway epithelial cells are implicated in different diseases, such as asthma ${ }^{22}$ and BPD. ${ }^{23}$ Environmental factors regulating epigenetic modifications are implicated in the pathogenesis of asthma. ${ }^{24}$ Epigenetic modification of SEMA3A was involved in the progression of breast cancer. ${ }^{25}$ The epigenetic modification of SEMA3A involved in the development of BPD must be investigated in the future research.

Inflammation is involved in the pathogenesis of BPD, and the anti-inflammatory effect of omega- 3 fatty acids prevented hyperoxic lung injury in newborn rats. ${ }^{26}$ Previous studies have demonstrated that SEMA3A reduced inflammation in mice with bronchial asthma ${ }^{27}$ and autoimmune arthritis. ${ }^{28}$ The present results have demonstrated that over-expression of SEMA3A attenuated hyperoxia-induced increased levels of IL-1 $\beta$, MCP-1, and TNF- $\alpha$ in the lung tissue of BPD rats. Moreover, NF-kB pathway was reported to be involved in SEMA3A-mediated immune dysfunction during lipopolysaccharide (LPS)-induced sepsis. ${ }^{29}$ Enhanced 
(A)
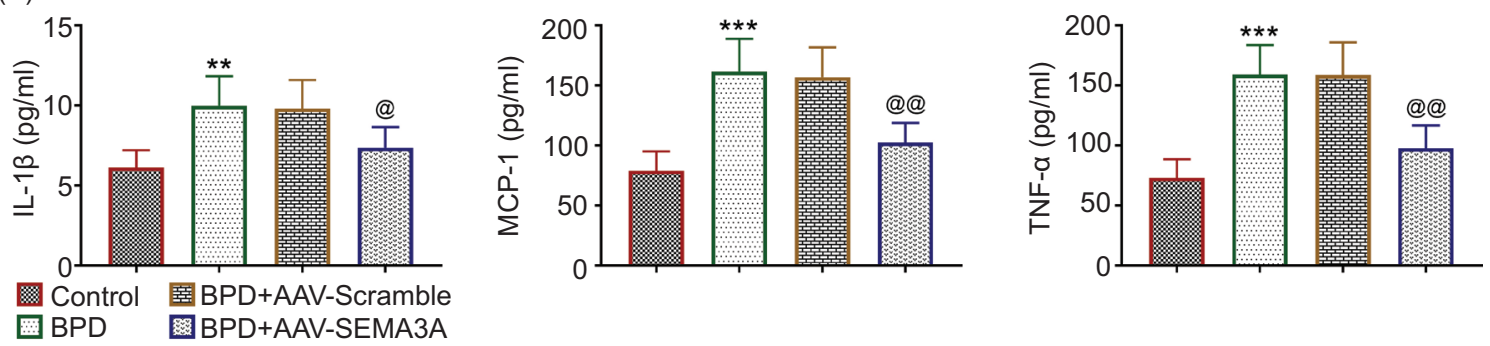

(B)
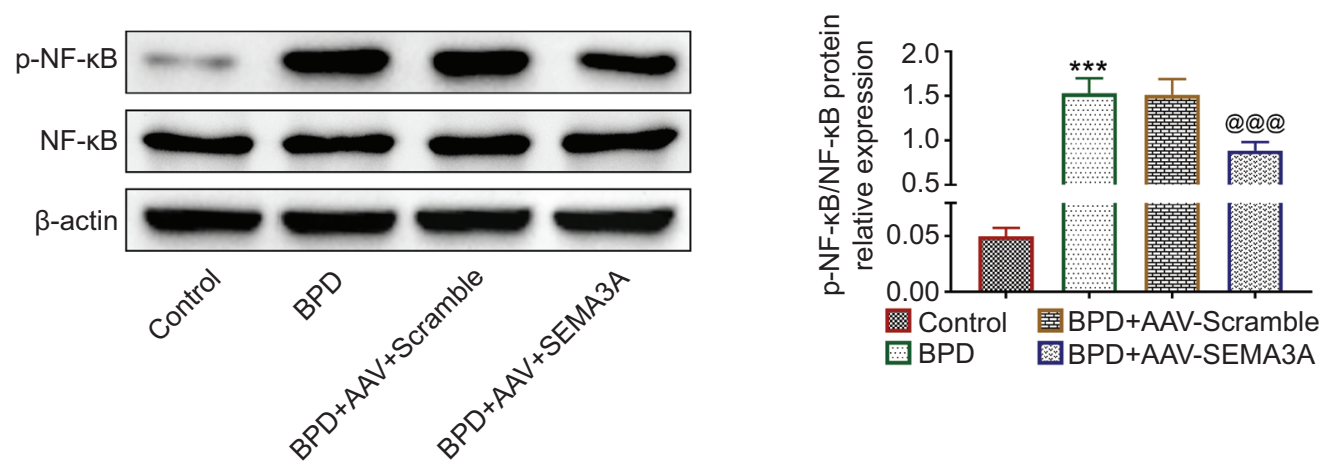

Figure 3 SEMA3A suppressed hyperoxia-induced inflammation in BPD rats. (A) AAV-SEMA3A injection attenuated hyperoxiainduced increased levels of IL-1 $\beta, M C P-1$, and TNF-a. (B) AAV-SEMA3A injection attenuated hyperoxia-induced increased expression

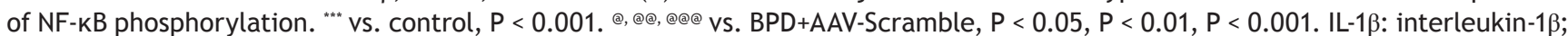
MCP-1: monocyte chemoattractant protein-1; TNF-a: tumor necrosis factor alpha; SEMA3A: semaphorin 3A.

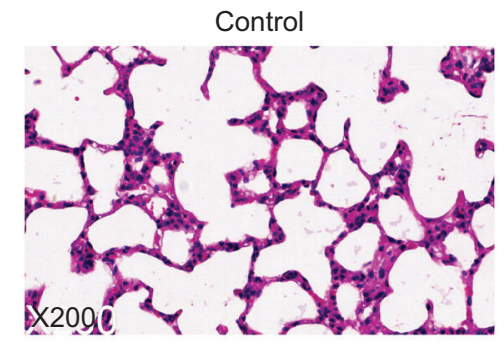

BPD+AAV-Scramble

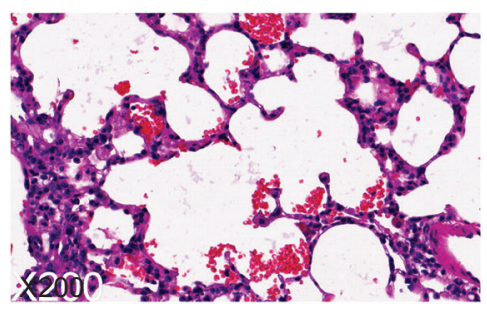

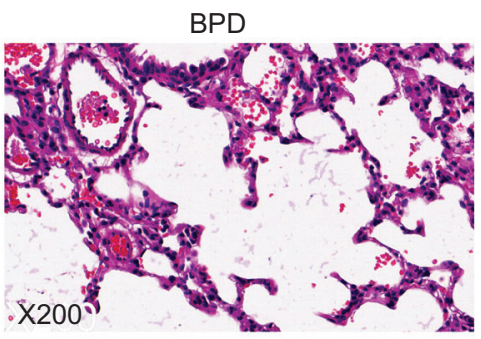

BPD+AAV-SEMA3A

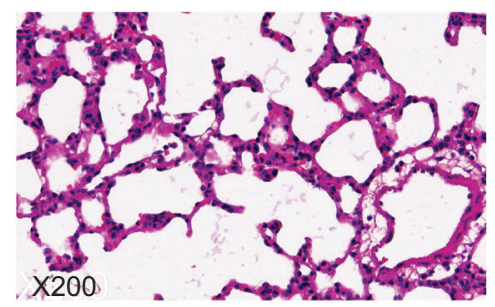

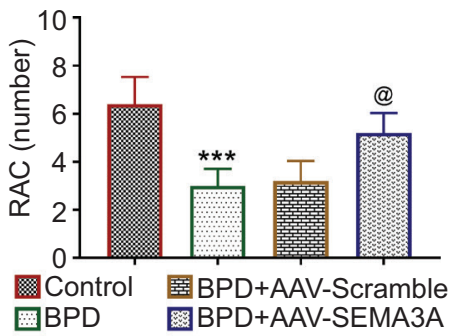

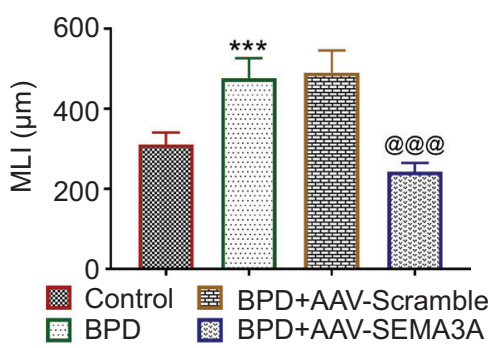

Figure 4 SEMA3A ameliorated hyperoxia-induced histopathological changes in BPD rats. AAV-SEMA3A injection ameliorated hyperoxia-induced alveolar injury, simplification, and inflammation, and attenuated hyperoxia-induced decreased number of RAC

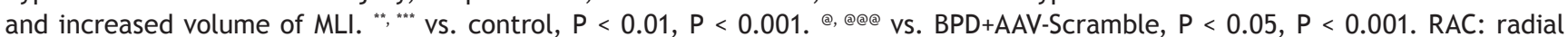
alveolar count; MLI: mean linear intercept; SEMA3A: semaphorin 3A.

protein expression of NF-kB phosphorylation in hyperoxia-induced rats was reduced by the over-expression of SEMA3A, suggesting that SEMA3A suppressed hyperoxia-induced inflammation in BPD rats through inactivation of NF-kB pathway.

ERK pathway is important for the growth and survival of epithelial cells in response to hyperoxia. ${ }^{30}$ ERK1/2 pathway was implicated in the proliferation and migration of lung fibroblasts in newborn rats, ${ }^{31}$ and endothelial ERK2 was identified as a potential target for BPD in infants. $^{32}$ Inhibition of ERK1/2 pathway contributed to the prevention of tetrandrine in hyperoxia-induced rats. ${ }^{5}$ ERK/JNK was known as a downstream signal pathway of SEMA3A, and SEMA3A suppressed the activation of ERK 

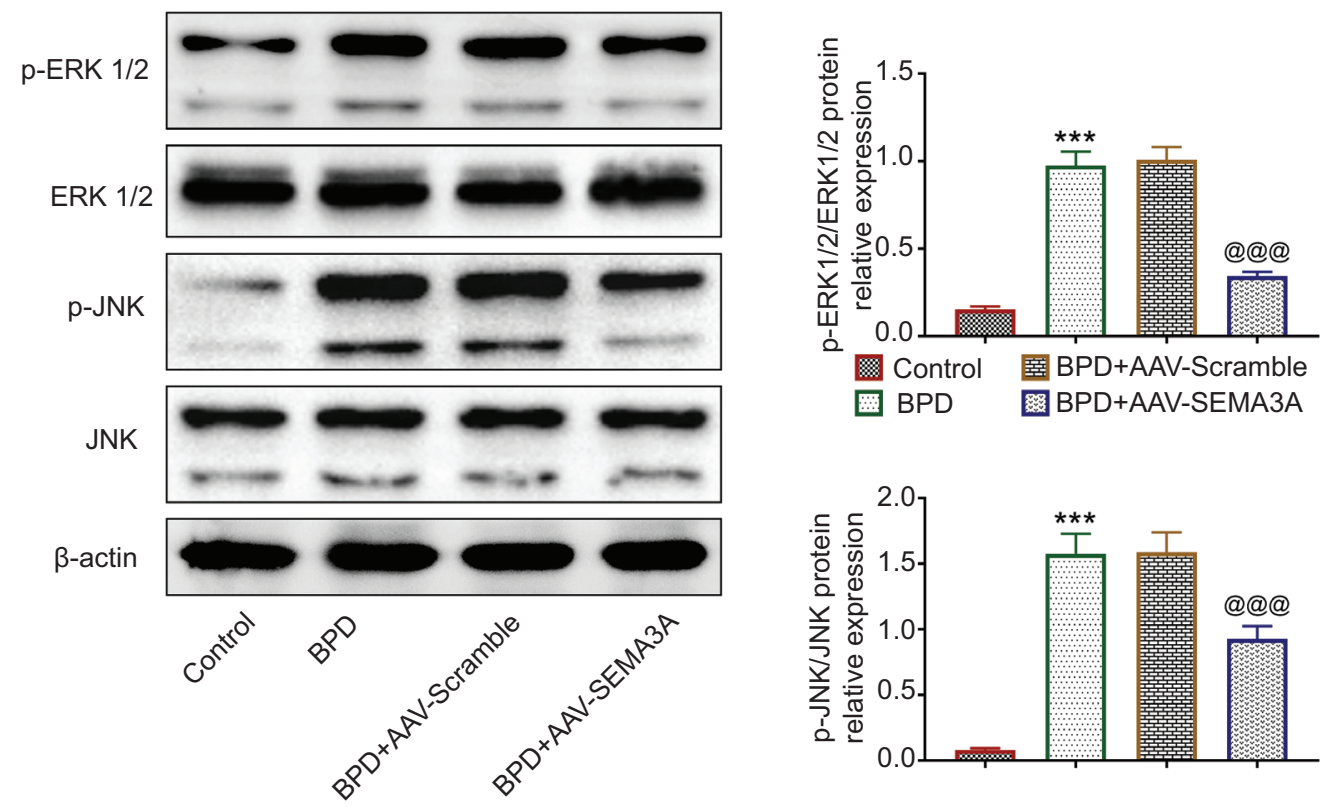

Figure 5 SEMA3A suppressed the activation of ERK/JNK pathway in BPD rats. AAV-SEMA3A injection attenuated hyperoxiainduced increased expression levels of ERK and JNK phosphorylation. "*** vs. control, $\mathrm{P}<0.001$. ®@® vs. BPD+AAV-Scramble, $\mathrm{P}<$ 0.001. SEMA3A: semaphorin 3A.

to inhibit excessive mechanical stress-induced inflammation in chondrocytes..$^{13}$ Here, results established that SEMA3A counteracted with the promotive effect of hyperoxia on the phosphorylated protein expressions of ERK1/2 and JNK, indicating that SEMA3A suppressed the activation of ERK/JNK pathway in hyperoxia-induced BPD rats. Oxidative stress caused alveolar epithelial cell injuries and contributed to the development of BPD. ${ }^{33}$ SEMA3A was involved in miR-203-3p-mediated oxidative stress of high glucose-induced mice podocytes. ${ }^{34}$ Taken together, SEMA3A might exert an anti-oxidant effect in hyperoxiainduced BPD rats.

\section{Conclusion}

SEMA3A exerted anti-apoptotic and anti-inflammatory effects against hyperoxia-induced lung injury in neonatal rats through inhibition of NF-KB and ERK1/2 pathways. Our findings provided evidence that SEMA3A might be a potential target for BPD. However, the effect of SEMA3A on alveolar epithelial cell proliferation and oxidative stress, as well as the pathway involved in SEMA3A-mediated cell apoptosis in hyperoxia-induced BPD rats, must be investigated in the future research.

\section{References}

1. Baud O, Maury L, Lebail F, Ramful D, El Moussawi F, Nicaise C, et al. Effect of early low-dose hydrocortisone on survival without bronchopulmonary dysplasia in extremely preterm infants (PREMILOC): A double-blind, placebo-controlled, multicentre, randomised trial. Lancet. 2016;387(10030):1827-36. https://doi.org/10.1016/S0140-6736(16)00202-6
2. Niver D. Bronchopulmonary dysplasia: Structural challenges and stem cell treatment potential. Adv Neonatal Care. 2014 Feb;14(1):E1-E11. https://doi.org/10.1097/ANC. 0000000000000050

3. Bach KP, Kuschel CA, Hooper SB, Bertram J, McKnight S, Peachey SE, et al. High bias gas flows increase lung injury in the ventilated preterm lamb. PLoS One. 2012;7(10):e47044. https://doi.org/10.1371/journal.pone.0047044

4. Pasha AB, Chen X-Q, Zhou G-P. Bronchopulmonary dysplasia: Pathogenesis and treatment. Exp Ther Med. 2018;16(6):431521. https://doi.org/10.3892/etm.2018.6780

5. Jiao B, Tang Y, Liu S, Guo C. Tetrandrine attenuates hyperoxia-induced lung injury in newborn rats via NF-kB p65 and ERK1/2 pathway inhibition. Ann Transl Med. 2020;8(16):1018. https://doi.org/10.21037/atm-20-5573

6. Kallet RH, Matthay MA. Hyperoxic acute lung injury. Respir Care. 2013;58(1):123-41. https://doi.org/10.4187/respcare.01963

7. Zhu Y, Fu J, Yang H, Pan Y, Yao L, Xue X. Hyperoxia-induced methylation decreases RUNX3 in a newborn rat model of bronchopulmonary dysplasia. Respir Res. 2015;16(1):75. https:// doi.org/10.1186/s12931-015-0239-x

8. Chen X, Zhang X, Pan J. Effect of montelukast on bronchopulmonary dysplasia (BPD) and related mechanisms. Med Sci Monit. 2019;25:1886-93. https://doi.org/10.12659/MSM.912774

9. Mo W, Li Y, Chang W, Luo Y, Mai B, Zhou J. The role of LncRNA $\mathrm{H} 19$ in MAPK signaling pathway implicated in the progression of bronchopulmonary dysplasia. Cell Transplant. 2020;29:963689720918294. https://doi. org $/ 10.1177 / 0963689720918294$

10. Toledano S, Nir-Zvi I, Engelman R, Kessler O, Neufeld G. Class-3 semaphorins and their receptors: Potent multifunctional modulators of tumor progression. Int J Mol Sci. 2019;20(3):556. https://doi.org/10.3390/ijms20030556

11. Higgins DMO, Caliva M, Schroeder M, Carlson B, Upadhyayula PS, Milligan BD, et al. Semaphorin 3A-mediated brain tumor stem cell proliferation and invasion in EGFRviii mutant gliomas. BMC Cancer. 2020;20(1):1213. https://doi.org/10.1186/ s12885-020-07694-4 
12. Nehil M, Paquette J, Tokuyasu T, McCormick F. High mobility group box 1 promotes tumor cell migration through epigenetic silencing of semaphorin 3A. Oncogene. 2014;33(44):5151-62. https://doi.org/10.1038/onc.2013.459

13. Sumi C, Hirose N, Yanoshita M, Takano M, Nishiyama S, Okamoto $\mathrm{Y}$, et al. Semaphorin $3 \mathrm{~A}$ inhibits inflammation in chondrocytes under excessive mechanical stress. Mediators Inflamm. 2018;2018:5703651. https://doi.org/10.1155/2018/5703651

14. Huang $B$, Zhang $Q$, Yuan $Y$, Xin N, He K, Huang $Y$, et al. Sema3a inhibits the differentiation of Raw264.7 cells to osteoclasts under $2 \mathrm{~Gy}$ radiation by reducing inflammation. PLoS One. 2018;13(7):e0200000. https://doi.org/10.1371/journal. pone. 0200000

15. Becker PM, Tran TS, Delannoy MJ, He C, Shannon JM, McGrath-Morrow S. Semaphorin 3A contributes to distal pulmonary epithelial cell differentiation and lung morphogenesis. PLoS One. 2011;6(11):e27449. https://doi.org/10.1371/ journal.pone.0027449

16. Joza S, Wang J, Fox E, Hillman V, Ackerley C, Post M. Loss of semaphorin-neuropilin-1 signaling causes dysmorphic vascularization reminiscent of alveolar capillary dysplasia. Am J Pathol. 2012;181(6):2003-17. https://doi.org/10.1016/j. ajpath.2012.08.037

17. Joza $\mathrm{S}$, editor The role of semaphorin-neuropilin-1 signalling in pulmonary vascular development. Canada: Toronto, 2012.

18. Vadivel A, Alphonse RS, Collins JJP, van Haaften T, O'Reilly M, Eaton $\mathrm{F}$, et al. The axonal guidance cue semaphorin $3 \mathrm{C}$ contributes to alveolar growth and repair. PLoS One. 2013;8(6):e67225. https://doi.org/10.1371/journal.pone.0067225

19. Zhang Y, Du H, Yu X, Zhu J. Fucoidan attenuates hyperoxiainduced lung injury in newborn rats by mediating lung fibroblasts differentiate into myofibroblasts. Ann Transl Med. 2020;8(22):1501. https://doi.org/10.21037/atm-20-6601

20. Ranganathan P, Jayakumar C, Mohamed R, Weintraub NL, Ramesh G. Semaphorin $3 \mathrm{~A}$ inactivation suppresses ischemia-reperfusion-induced inflammation and acute kidney injury. Am J Physiol Renal Physiol. 2014;307(2):F183-94. https://doi.org/10.1152/ajprenal.00177.2014

21. Yun-Jia L, Xi C, Jie-Qiong Z, Jing-Yi Z, Sen L, Jian $Y$. Semaphorin3A increases M1-like microglia and retinal ganglion cell apoptosis after optic nerve injury. Cell Biosci. 2021;11(1):97. https://doi.org/10.1186/s13578-021-00603-7

22. Potaczek DP, Miethe S, Schindler V, Alhamdan F, Garn H. Role of airway epithelial cells in the development of different asthma phenotypes. Cell Signal. 2020;69:109523. https://doi. org/10.1016/j.cellsig.2019.109523

23. Roos $A B$, Berg $T$, Nord $M$. A relationship between epithelial maturation, bronchopulmonary dysplasia, and chronic obstructive pulmonary disease. Pulm Med. 2012;2012:196194. https://doi.org/10.1155/2012/196194

24. Alizadeh Z, Mortaz E, Adcock I, Moin M. Role of epigenetics in the pathogenesis of asthma. Iran J Allergy Asthma Immunol. 2017:82-91. PMID: 28601047
25. Beetch M, Lubecka K, Shen K, Flower K, Harandi-Zadeh S, Suderman $M$, et al. Stilbenoid-mediated epigenetic activation of semaphorin $3 \mathrm{~A}$ in breast cancer cells involves changes in dynamic interactions of DNA with DNMT3A and NF1C transcription factor. Mol Nutrit Food Res. 2019;63(19):1801386. https://doi.org/10.1002/mnfr.201801386

26. Kul M, Gönül E, Akcan AB, Küçükodacı Z, Süleymanoğlu S. The effects of omega-3 fatty acids on the newborn rat hyperoxic lung injury. J Matern Fetal Neonatal Med. 2020;33(14):243440. https://doi.org/10.1080/14767058.2018.1554042

27. Adi SD, Eiza N, Bejar J, Shefer H, Toledano S, Kessler O, et al. Semaphorin $3 \mathrm{~A}$ is effective in reducing both inflammation and angiogenesis in a mouse model of bronchial asthma. Front Immunol. 2019;10:550. https://doi.org/10.3389/ fimmu.2019.00550

28. Catalano A. The neuroimmune semaphorin-3a reduces inflammation and progression of experimental autoimmune arthritis. J Immunol. 2010;185(10):6373. https://doi.org/10.4049/ jimmunol.0903527

29. Gao Y, Wang C, Wang Z, Li W, Liu Y, Shou S, et al. Semaphorin $3 \mathrm{~A}$ contributes to sepsis-induced immunosuppression by impairing CD4<sup $>+</$ sup $>\mathrm{T}$ cell anergy. Mol Med Rep. 2021;23(4):302. https://doi.org/10.3892/mmr.2021.11941

30. Zaher TE, Miller EJ, Morrow DMP, Javdan M, Mantell LL. Hyperoxia-induced signal transduction pathways in pulmonary epithelial cells. Free Radic Biol Med. 2007;42(7):897-908. https://doi.org/10.1016/j.freeradbiomed.2007.01.021

31. Hu Y, Fu J, Liu X, Xue X. ERK1/2 signaling pathway activated by EGF promotes proliferation, transdifferentiation, and migration of cultured primary newborn rat lung fibroblasts. BioMed Res Int. 2020;2020:7176169. https://doi. org/10.1155/2020/7176169

32. Menon RT, Shrestha AK, Barrios R, Reynolds C, Shivanna B. Tie-2 cre-mediated deficiency of extracellular signal-regulated kinase 2 potentiates experimental bronchopulmonary dysplasia-associated pulmonary hypertension in neonatal mice. Int J Mol Sci. 2020;21(7):2408. https://doi.org/10.3390/ ijms21072408

33. Wu D, Liang M, Dang H, Fang F, Xu F, Liu C. Hydrogen protects against hyperoxia-induced apoptosis in type II alveolar epithelial cells via activation of PI3K/Akt/Foxo3a signaling pathway. Biochem Biophy Res Commun. 2018;495(2):1620-7. https://doi. org/10.1016/j.bbrc.2017.11.193

34. Chen J, Xu Q, Zhang W, Zhen Y, Cheng F, Hua G, et al. MiR203-3p inhibits the oxidative stress, inflammatory responses and apoptosis of mice podocytes induced by high glucose through regulating Sema3A expression. Open Life Sci. 2020;15(1):939-50. https://doi.org/10.1515/biol-2020-0088 